\title{
Laparoscopic Hiatal Herniorrhaphy in a Large Hiatal Hernia as a Rare Cause of Dyspnea and Anemia: A Case Report
}

\author{
Masood Amini ${ }^{1,2,{ }^{*}}$, Mohammadhassan Hashemizadeh ${ }^{1,2}$, Hadi Hadavi ${ }^{1,2}$, Masood Sepehrimanesh ${ }^{3}$ \\ and Maryam Setoodeh ${ }^{2}$ \\ ${ }^{1}$ Laparoscopy Research Center, Shiraz University of Medical Sciences, Shiraz, IR Iran \\ ${ }^{2}$ Obesity Specialized Clinics, Mother and Child Specialized Hospital, Shiraz, IR Iran \\ ${ }^{3}$ Gastrointestinal and Liver Diseases Research Center, Guilan University of Medical Sciences, Rasht, Iran \\ "Corresponding author: Laparoscopy Research Center, Shiraz University of Medical Sciences, Shiraz, Iran. Tel: +98-71-32279711, Email: shirazobesity@sums.ac.ir \\ Received 2016 May 16; Revised 2016 June 17; Accepted 2016 July 31.
}

\begin{abstract}
Introduction: Herniation of the stomach through the esophageal hiatus into the thoracic cavity is a common affliction in modern humans. Its incidence in North America has ranged from 10\% to $80 \%$. However, approximately complete herniation of the stomach is scarce. Therefore, we discussed this unusual case.

Case Presentation: A 43-year-old man with a hypochromic, microcytic, iron-deficient anemia picture, postprandial and postexercise respiratory failures, and epigastric pain was examined by a gastroenterologist in the outpatient clinic of mother and child specialized hospital in Shiraz, Iran in November 2015. Left thorax hemiatrophy was obvious upon physical examination. Positive Helicobacter pylori, peripyloric erythema, and mild chronic active gastritis were diagnosed after an upper gastrointestinal (GI) endoscopy and colonoscopy. In a barium-swallow upper GI series, malrotation of most of the patient's stomach and the stomach's dislocation in to the thoracic cavity were detected. A CT scan also showed a large hiatus hernia with a large part of the stomach inserted in the defect. The patient revealed a history of a motorcycle accident about 28 years earlier. After the administration of general anesthesia and the semilateral positioning of the patient, five ports were inserted (three $5 \mathrm{~mm}$ and two $10 \mathrm{~mm}$ ). The stomach was relocated into the abdominal cavity, and the bloody sac was dissected from the left and then the right cross, detached from the thoracic cavity, and completely removed. Two cruses were sutured together using a 1/0 silk suture, and after releasing the short gastrics, a prophylactic Dor fundoplication was performed. The next morning, oral nutrition was started, and the patient was discharged in a good general health condition.

Conclusions: Laparoscopic repair of a large hiatus hernia plus Dor fundoplication is an effective method of treatment of such a defect without any symptoms. However, it may be associated with the mild to moderate prevalence of recurring hernias.
\end{abstract}

Keywords: Accidents, Anemia, Hernia, Hiatal, Laparoscopy

\section{Introduction}

A hiatal hernia is defined as an abnormal protrusion of the stomach with another intra-abdominal organ that occurs, in some cases, above the diaphragm from the esophageal hiatus (1). Hernias of the esophageal hiatus are classified according to the position of the gastroesophageal junction (GEJ) and the extent of the herniated stomach $(2,3)$ as either type I (sliding hiatal hernias), in which the GEJ migrates above the diaphragm and the stomach remains in its usual longitudinal alignment; type II (pure paraesophageal hernias [PEH]), in which the GEJ remains in its normal anatomic position but a portion of the fundus herniates through the diaphragmatic hiatus adjacent to the esophagus; type III (a combination of types I and II); or type IV, which is characterized by the presence of a structure other than the stomach, such as the omentum, colon, or small bowel, within the hernia sac.

The prevalence of hiatal hernias in upper gastrointestinal (GI) endoscopy series was reported as $0.8 \%-2.9 \%$ (3). While some patients with PEHs are asymptomatic, most PEHs cause mild symptoms, and the underlying condition is generally only detected on a chest radiography for other reasons (4). Type I hernias may be associated with gastroesophageal reflux disease, and its related symptoms include heartburn, epigastric pain, and Barrett's epithelium (5). Additionally, mild and acute obstructive symptoms may be seen in type I hernia patients (6). Dysphagia, postprandial fullness, and upper abdominal pain occur in these patients, but the pain is often relieved by vomiting (2). Such cases have been reported as postoperative complications 
(7). This report discusses a rare case of a PEH that was repaired by laparoscopic surgery in a patient without any previous history of open or laparoscopic surgeries.

\section{Case Presentation}

A 43-year-old man presented to the outpatient clinic of mother and child specialized hospital in Shiraz, Iran in November 2015 with increasing fatigue and shortness of breath lasting for over one year. His demographic information included a height of $178 \mathrm{~cm}$, a weight of $85 \mathrm{~kg}$, a body mass index of $26.82 \mathrm{~kg} / \mathrm{m}^{2}$, and systolic and diastolic blood pressures of 120 and $80 \mathrm{mmHg}$, respectively. The patient noted that his shortness of breath was aggravated with exertion and after the ingestion of food. The patient did not describe an underlying chronic disease. He had a history of a motorcycle accident about 28 years ago.

Upon the physical examination, the patient's vital signs were stable. The physical examination found kyphosis and hypertrophy of the Lt. thorax. Hematological variables were assessed using a Sysmex KX-21N Hematology Analyzer (USA). The patient's iron concentration and total iron-binding capacity (TIBC) were measured using a BIOLIS 24i Premium biochemical autoanalyzer (Japan). His ferritin was assessed by ELISA (Monobind, USA), and hemoglobin $(\mathrm{Hb})$ electrophoresis was performed using a SAIO electrophoresis instrument (Italia). The following results were obtained: white blood cell, 9200/ $\mathrm{mm}^{3}$; $\mathrm{Hb}, 8.4 \mathrm{~g} / \mathrm{dL}$; mean corpuscular volume, $65.8 \mathrm{fL}$; platelet, $507000 / \mathrm{mm}^{3}$; iron, $48 \mu \mathrm{g} / \mathrm{dL}$; TIBC, $356 \mu \mathrm{g} / \mathrm{dL}$; ferritin, $1.72 \mathrm{ng} / \mathrm{mL} ; \mathrm{Hb}$ A, 98.1\%; Hb A2, 1.9\%. A fecal occult blood test was positive. The patient's biochemical parameters were within a normal range.

On a posterioanterior chest radiograph, increased cardiothoracic index, enlarged mediastinum, and a mass appearance with an air-fluid level superposed with cardiac contours were observed. An abdominopelvic computed tomography (CT) scan that included the axial and coronal sections showed a large hiatal hernia with an enlarged part of the stomach in the defect (Figure 1).

On the upper GI endoscopy, only prepyloric erythema was found, and mild chronic gastritis and Helicobacter pylori were shown. The results of a colonoscopy, which was performed to determine the etiology of the patient's iron deficiency anemia, were considered normal. On the barium-swallow upper GI series, the patient's stomach showed a degree of malrotation, and most of the stomach was located in the left thorax (Figure 2).

The patient's hemogram was normal three months after iron replacement therapy and the patient was a candidate for operation. In the operation room, after planning and design and placing the patient under general anesthesia, with the patient in a supine position, five ports were inserted in the upper abdomen to enter the abdominal cavity. Exploration was done with an intra-abdominal pressure of $13 \mathrm{mmHg}$. Nearly all of the visible stomach and omentum were herniated into a large hiatal hernia. In a steep reverse Trendelenburg position, the omentum and stomach were reduced to the abdomen with three atraumatic graspers. The excision of the peritoneal hernia sac from the mediastinum was performed via dissection from the right crus to the left crus without injury to the pleura and the vagus nerves. After the anterior, medial, and posterior dissections were accomplished and after the circumferential mobilization of the lower esophagus and upper stomach, the crural defect was repaired with a silk1 suture without tension. After the release of short gastric vessels on the fundus, Dor fundoplication was performed. During the operation, a spleen injury occurred that was controlled with surgicele and cautery (Figure 3 ).

In the postoperative course, the patient's control of gagging, belching, and vomiting was tested. The patient was not suffering from dysphasia or dyspnea. The postoperative chest X-ray did not show a pneumothorax. The patient tolerated a clear liquid diet on the first postoperative day. At the time of his discharge, the patient was placed on a soft diet. The patient's dyspnea was cured completely one month after surgery. He suffered from heartburn. A barium swallow showed gastritis and active ulcers in the greater curvature side of the gastric body and a mild hiatal hernia. Therefore, PPIs and anti-H. pylori drugs were started.

\section{Discussion}

Most cases of hiatal hernia are acquired rather than congenital (2) and most often occur as a postoperative complication. However, this case had no previous history of surgery, and only an anemic feature and a previous accident were recorded.

Although the standard technique for the repair of a $\mathrm{PEH}$ is a laparoscopic approach, transabdominal open repair may be most appropriate in emergency cases with peritoneal contamination or gastric necrosis (2). The sliding type of hiatal hernia, in which the GEJ slides together with a part of the stomach, is the most commonly observed type (8). Although the cause of the development of hiatal hernia is unknown, its incidence increases with advancing age (2). Relaxation at the diaphragmatic crura resulting from the aging process is accepted as the cause of the observation of more frequent and larger hiatal hernias in the elderly population (9). In this study, the patient was young, so the cause of his hiatal hernia may be related to his job or previous trauma. GI bleeding related to ulcer or erosion, iron deficiency anemia, mucosal prolapse, incarceration, 

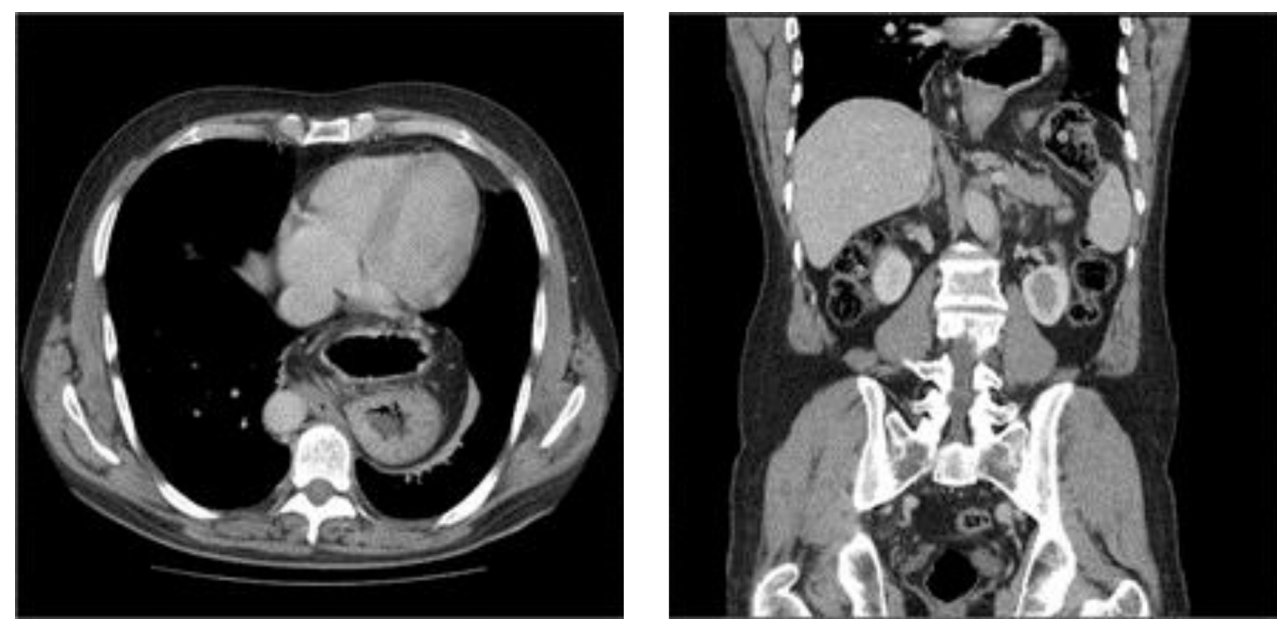

Figure 1. Axial and Coronal Images of the Hiatal Hernia on an Abdominopelvic CT

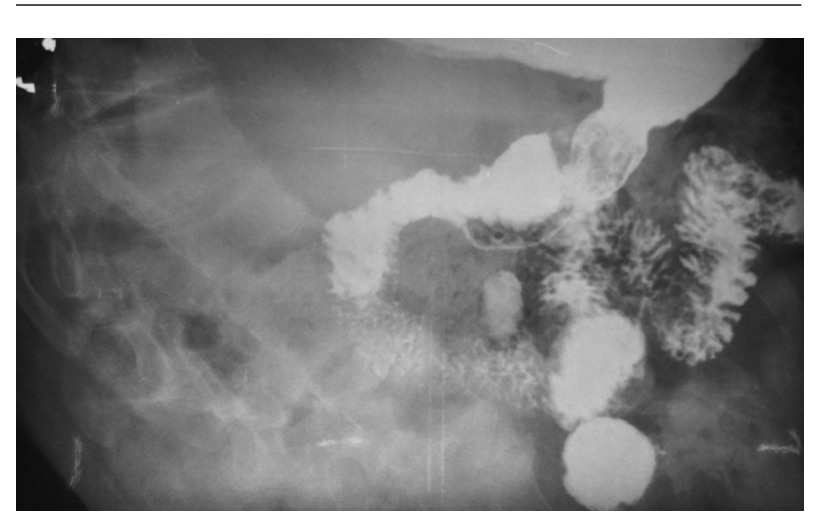

Figure 2. Barium-Swallow Radiograph

and volvulus are the main complications of hiatal hernia (8).

Patients with large diaphragmatic hiatal hernias occasionally manifest severe iron deficiency anemia (10). The association between iron deficiency anemia and hiatal hernia has been known. The main reason for this association is the hemorrhage resulting from linear ulcers and erosions (Cameron lesions) at mucosal folds where diaphragmatic compression occurs $(11,12)$, which is seen in $5.2 \%$ of patients with hiatal hernias who undergo esophagogastroduodenoscopy examinations. The prevalence of Cameron lesions seems to depend on the size of the hernia sac, with an increased prevalence with a larger hernia sac. In about two-thirds of cases, multiple Cameron lesions are noted rather than a solitary erosion or ulcer (13).

Another reason for iron deficiency anemia in hiatal hernia patients is chronic gastritis and its consequences.
Today, although they are often missed, these lesions, one of the occult reasons for both GI bleeding and iron deficiency anemia, are reported in $5 \%$ of patients with a hiatal hernia and $20 \%$ of patients with persistent anemia and recurrent bleeding (10). One study stated that Cameron lesions were not an uncommon cause of chronic GI bleeding and should be kept in mind when treating patients with iron deficiency anemia (14). In our case, the etiology of the patient's anemia may be due to chronic gastritis, but in the postoperative barium swallow, a Cameron lesion was seen that was not seen in the periupper endoscopy. Although a large hiatal hernia may cause iron deficiency anemia even without Cameron erosions (15), one of the most commonly missed lesions while performing an upper endoscopy in the work-up of iron deficiency anemia is a Cameron lesion located at the neck of a large hiatal hernia (16). Cameron lesions should be considered in any patient in whom a hiatal hernia is noted during an endoscopic examination (13). However, the patient's severe anemia was cured before surgery in the presented case. Mechanical trauma, ischemia, and acid mucosal injury may play a role in the pathogenesis of Cameron lesions. The choice of therapy to treat Cameron lesions, whether medical or surgical, should be individualized for each patient. Surgery in combination with proton pump inhibitor therapy is no better than proton pump inhibitor therapy alone for preventing the recurrence of anemia (15).

Although large hiatal hernias are infrequent, they can lead to atypical symptoms, such as chest pain and dyspnea, and rare complications, such as pulmonary edema and cardiac failure, due to the extent of the hernia and compression of the heart and pulmonary veins by organs protruded into the thorax cavity. Sahin et al. reported that a 

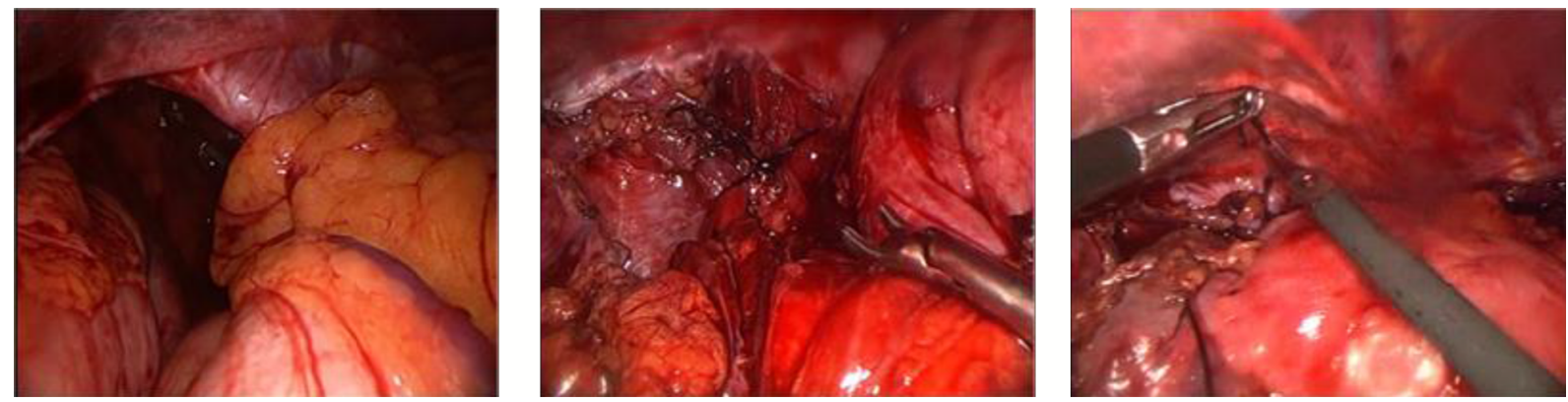

Figure 3. The Hiatal Defect (Left), the Laparoscopic Surgical Procedure (Mid), and the Final Closure of the Defect (Right)

large hiatal hernia caused cardiac failure through the compression to the left atrium in a case that presented with recurring acute cardiac failure attacks (3). In another case report, Chau et al. demonstrated a large hiatal hernia as the cause of chest pain in a patient that presented to an emergency department with acute angina (17). A hiatal hernia can manifest as a left atrial mass on echocardiography. A hiatal hernia can cause pulmonary edema and cardiac failure through pulmonary venous obstruction (16). In our case, chronic fatigue and exertion dyspnea particularly were aggravated during the postprandial period. The aggravation of dyspnea after heavy meals is thought to be due to pulmonary congestion from compression of the left atrium and the right pulmonary vein. Large hiatal hernias can be repaired either transabdominally (open or laparoscopic) or via a LT thoracotomy.

Large hiatal hernias can lead to cardiac symptoms and complications due to compression and iron deficiency anemia. The laparoscopic repair of large hiatal hernias plus Dor fundoplication is an effective method of treatment of such a defect. However, it may be associated with mild to moderate prevalence of recurrent hernia.

\section{Footnote}

Authors' Contribution: Masood Amini, Mohammadhassan Hashemizadehand and Hadi Hadavi visited, managed, and operated the case; Masood Sepehrimanesh was the coordinator of the group, wrote the manuscript, and finalized the manuscript; Maryam Setoodeh was the supervisor of the VIP department of the hospital and managed the patient after surgery; All authors had significant roles in the preparation of documents and providing the first draft.

\section{References}

1. Rezailashkajani M, Roshandel D, Shafaee S, Zali MR. High prevalence of reflux oesophagitis among upper endoscopies of Iranian patients. Eur J Gastroenterol Hepatol. 2007;19(6):499-506. doi: 10.1097/MEG.0b013e32811ebfec. [PubMed: 17489061].
2. Kohn GP, Price RR, DeMeester SR, Zehetner J, Muensterer OJ, Awad Z, et al. Guidelines for the management of hiatal hernia. Surg Endosc. 2013;27(12):4409-28. doi: 10.1007/s00464-013-3173-3. [PubMed: 24018762].

3. Sahin C, Akin F, Cullu N, Ozseker B, Kirli I, Altun I. A large intraabdominal hiatal hernia as a rare cause of dyspnea. Case Rep Cardiol. 2015;2015:546395. doi: 10.1155/2015/546395. [PubMed: 26229693].

4. Michel JM, Krahenbuhl L. Atlas of upper gastrointestinal and hepatopancreato-biliary surgery. Springer; 2016. p. 283-90.

5. Samakar K, McKenzie T], Tavakkoli A, Vernon AH, Robinson MK, Shikora SA. The effect of laparoscopic sleeve gastrectomy with concomitant hiatal hernia repair on gastroesophageal reflux disease in the morbidly obese. Obesity Surg. 2016;26(1):61-6.

6. Oleynikov D, Jolley JM. Paraesophageal hernia. Surg Clin North Am. 2015;95(3):555-65. doi: 10.1016/j.suc.2015.02.008. [PubMed: 25965129].

7. Kanamori J, Fujita T, Daiko H. Diaphragmatic herniation after thoracolaparoscopic esophagectomy for carcinoma of the esophagus: a report of six cases. Esophagus. 2016;13(1):88-92.

8. Kahrilas PJ, Pandolfino JE. Hiatus hernia. GI Motility online. 2006.

9. Noth I, Zangan SM, Soares RV, Forsythe A, Demchuk C, Takahashi SM, et al. Prevalence of hiatal hernia by blinded multidetector CT in patients with idiopathic pulmonary fibrosis. Eur RespirJ. 2012;39(2):344-51. doi: 10.1183/09031936.00099910. [PubMed: 21737563].

10. Moskovitz M, Fadden R, Min T, Jansma D, Gavaler J. Large hiatal hernias, anemia, and linear gastric erosion: studies of etiology and medical therapy. Am J Gastroenterol. 1992;87(5):622-6. [PubMed: 1595651].

11. Mobley JE, Christensen NA. Esophageal hiatal hernia: prevalence, diagnosis and treatment in an American city of 30,000. Gastroenterology. 1956;30(1):1-11. [PubMed: 13285670].

12. Roy RR, Sagar S, Bunch TJ, Aman W, Crusan DJ, Srivathsan K, et al. Hiatal Hernia is associated with an increased prevalence of atrial fibrillation in young patients. Hypertension. 2013;49:40.

13. Weston AP. Hiatal hernia with cameron ulcers and erosions. Gastrointest Endosc Clin NAm. 1996;6(4):671-9. [PubMed: 8899401].

14. Kimer N, Schmidt PN, Krag A. Cameron lesions: an often overlooked cause of iron deficiency anaemia in patients with large hiatal hernias. BMJ Case Rep. 2010;2010. doi: 10.1136/bcr.06.2010.3129. [PubMed: 22791730].

15. Panzuto F, Di Giulio E, Capurso G, Baccini F, D’Ambra G, Delle Fave G, et al. Large hiatal hernia in patients with iron deficiency anaemia: a prospective study on prevalence and treatment. Aliment Pharmacol Ther. 2004;19(6):663-70. doi: 10.1111/j.1365-2036.2004.01894.x. [PubMed: 15023168].

16. Pauwelyn KA, Verhamme M. Large hiatal hernia and iron deficiency anaemia: clinico-endoscopical findings. Acta Clin Belg. 2005;60(4):166-72. doi: 10.1179/acb.2005.030. [PubMed:16279396].

17. Chau B, Dufel S. Gastric volvulus. Emerg Med J. 2007;24(6):446-7. doi: 10.1136/emj.2006.041947. [PubMed: 17513555]. 